\title{
Structural Transformation upon Nitrogen Doping of Ultrananocrystalline Diamond Films by Microwave Plasma CVD
}

\author{
Chien-Chung Teng, ${ }^{1}$ Shin-Min Song, ${ }^{2}$ Chien-Min Sung, ${ }^{3}$ and Chhiu-Tsu Lin ${ }^{1}$ \\ ${ }^{1}$ Department of Chemistry and Biochemistry, Northern Illinois University, Dekalb, IL 60115, USA \\ ${ }^{2}$ Department of Mechanical Engineering, Northern Illinois University, Dekalb, IL 60115, USA \\ ${ }^{3}$ Diamond Technology Center, Kinik Company, No. 64, Chung-San Road, Ying-Ko, Taipei County 239, Taiwan
}

Correspondence should be addressed to Chhiu-Tsu Lin, ctlin@niu.edu

Received 31 October 2008; Revised 16 January 2009; Accepted 11 February 2009

Recommended by Rakesh Joshi

\begin{abstract}
The molecular properties and surface morphology of undoped and N-doped ultra-nanocrystalline diamond (UNCD) films deposited by microwave plasma CVD with addition of nitrogen are investigated with various spectroscopic techniques. The results of spatially resolved Raman scattering, ATR/FT-IR and XPS spectra show more amorphous and $\mathrm{sp}^{2} / \mathrm{sp}^{3}$ ratio characteristics in N-doped UNCD films. The surface morphology in AFM scans shows larger nanocrystalline diamond clusters in N-doped UNCD films. Incorporation of nitrogen into UNCD films has promoted an increase of amorphous $\mathrm{sp}^{2}$-bonded carbons in the grain boundaries and the size of nanocrystalline diamond grains that are well correlated to the reported enhancement of conductivity and structural changes of UNCD films.
\end{abstract}

Copyright (C) 2009 Chien-Chung Teng et al. This is an open access article distributed under the Creative Commons Attribution License, which permits unrestricted use, distribution, and reproduction in any medium, provided the original work is properly cited.

\section{Introduction}

Ultrananocrystalline diamond (UNCD) films have become very attractive materials for microelectronics applications since UNCD films with and without nitrogen doping have recently been shown to have mild $\mathrm{n}$ - and p-type semiconductor characteristics [1-8]. UNCD films are usually prepared by $1 \% \mathrm{CH}_{4} / \mathrm{Ar}$ microwave plasma CVD (MPCVD) [9]. Typically, UNCD films compose of $3 \sim 8 \mathrm{~nm}$ polycrystalline nanodiamond grains, containing the mixtures of $\mathrm{sp}^{2} / \mathrm{sp}^{3}$-bonded carbon (trans-polyacetylene (t-PA) like, and graphite-like $\mathrm{sp}^{2}$-bonded $\mathrm{CH}$ groups and $\mathrm{sp}^{3}$-bonded $\mathrm{CH}_{2}$ groups), with boundaries of $\sim 0.5 \mathrm{~nm}$ in width $[3,10-12]$.

Both theoretical calculations and experimental data showed that the preferential incorporation of nitrogen into the grain boundaries of N-doped UNCD films would form the larger clustering and disordering of graphite-like $\mathrm{sp}^{2}$ bonded carbons $[5,6,11]$. Recently, diamond nanowires consisting of diamond core sheathed with $\mathrm{sp}^{2}$-bonded carbon about $1 \mathrm{~nm}$ in thickness are observed in N-doped UNCD films with addition of $10 \% \mathrm{~N}_{2}[11,12]$.

Raman spectroscopy has been used to characterize molecular properties of UNCD films. On the other hand,
IR spectroscopy is known as a complementary technique to Raman scattering for the studies of structural vibrations, such as $\mathrm{C}-\mathrm{H}$ bonds stretching $\left(2750 \sim 3300 \mathrm{~cm}^{-1}\right)$ in sp $\mathrm{sp}^{2} / \mathrm{sp}^{3}$ carbon-based materials $[2,13]$. In particular, attenuated total reflection Fourier transform infrared (ATR/FT-IR) spectroscopy is an effective technique for UNCD mirrorflat thin films samples due to the increasing optical path length by the multiple-reflected radiation in the total internal reflection element [14]. In this paper, we investigate and compare the spatial uniformity of molecular properties and surface morphology of MPCVD UNCD films processed with and without the addition of $20 \%$ nitrogen by using Raman and ATR/FT-IR spectroscopy as well as AFM for morphology images and XPS for chemical environment identifications. The correlations of spectroscopic results and the reported electrical conductivity and structural modifications are also discussed.

\section{Experimental}

2.1. UNCD Films by MPCVD. The UNCD films grown on $\mathrm{Si}$ with and without $\mathrm{N}_{2}$ addition samples were prepared using 6 
in CYRRANUS Innovative Plasma System (MPECVD, IPLAS $\mathrm{GmbH}$ ) and provided by Dr. Gruen's group at Argonne National Lab, USA. The details of deposition parameters were the same as described in [12].

The UNCD films were deposited on a large 4 in Si wafer. The size, distribution, and uniformity of microwave plasma and substrate temperature can effectively affect the quality, property, and morphology of UNCD films. In particular, the UNCD film grown at the center of the Si wafer may be quite different from that at the edge. In this work, the UNCD film on Si wafer was divided into ten (10) intervals along the radius from the edge to the center, and labeled as \#1 $\rightarrow$ \#10.

2.2. Characterization Methods. Renishaw Raman Scattering Noodles System 2000, equipped with a Leica microscope and a HeNe red laser $(632.8 \mathrm{~nm})$ as an excitation source, was used to examine the molecular properties of UNCD films. X-ray photoelectron spectroscopy $\left(\mathrm{Mg}-\mathrm{K}_{\alpha}\right.$ radiation of $1253.6 \mathrm{eV}$ with a surface analysis depth of $\sim 2 \mathrm{~nm}$ ) was used to prove the binding states of carbon $1 \mathrm{~s}$ core level in UNCD films without $\mathrm{Ar}^{+}$sputtering pretreatment. ATR/FT-IR spectrometer (PIKE, MIRacle Single Reflection Horizontal ATR Accessory equipped with $1.8 \mathrm{~mm}$ round ZnSe crystal IRE plate) was used to diagnose the molecular vibrations of UNCD films in mid-IR $\left(650 \sim 4000 \mathrm{~cm}^{-1}\right)$ range recorded with 500 scans and $1 \mathrm{~cm}^{-1}$ resolution. Atomic force microscopy (AFM, Quesant Q-scope 350) measurements were scanned for the surface morphology of UNCD films.

\section{Results and Discussions}

3.1. Raman Spectra of UNCD Films. The spectroscopic measurement was monitored at each interval position. Figure 1 shows Raman spectra of undoped and N-doped UNCD films. The top two (green and pink) and the bottom two (brown and blue) are recorded at the edge and at the center of substrate, respectively. The assignment and interpretation of Raman spectra of undoped and $\mathrm{N}$-doped UNCD films deposited from $1 \% \mathrm{CH}_{4} / \mathrm{N}_{2} / \mathrm{Ar}$ MPCVD have been extensively discussed recently $[4,6,11,12]$. The following spectral features are typically observed: (1) a broad band at $1332 \mathrm{~cm}^{-1}$ from nanodiamond crystallites, (2) the $\mathrm{sp}^{2}$-bonded carbon features around $1340 \sim 1600 \mathrm{~cm}^{-1}$ (in particular at $\sim 1350 \mathrm{~cm}^{-1}$ and $\sim 1580 \mathrm{~cm}^{-1}$ for graphite D\& G-bands), and (3) The C-H vibration characteristics of short-chain conjugated t-PA in the grain boundaries at 1120 $\sim 1190 \mathrm{~cm}^{-1}$ together with a weak peak at $1460 \mathrm{~cm}^{-1}$ and the overtone features at $2245 \sim 2810 \mathrm{~cm}^{-1}[3,9,10]$. Moreover, the $\mathrm{sp}^{3} / \mathrm{sp}^{2}$ ratio in N-doped UNCD films normally decreases as compared to the undoped UNCD films [4].

In the bottom two spectra of Figure 1, the differences in the Raman spectra between undoped and N-doped UNCD films taken at the center position are similar to those observed by Polyakov et al. [7]. However, Polyakov et al. did not report the Raman spectra measured at the edge position for UNCD films. When the top two spectra (edge) and bottom two spectra (center) in Figure 1 are compared, the following observations are noted: (1) a large increase (or

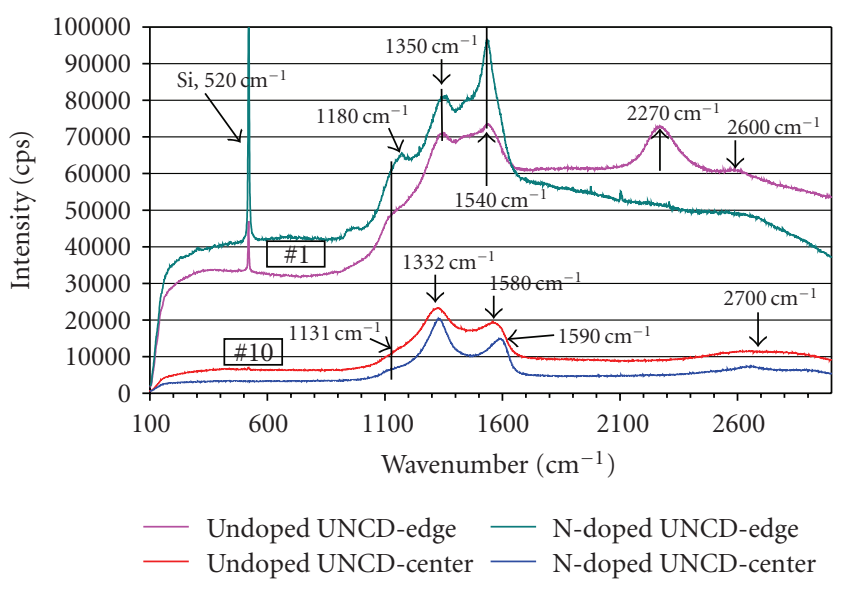

FIGURE 1: Raman spectra of UNCD films taken along the radius from the edge to the center position, labeled as \#1 $\rightarrow$ \#10.

almost inverse) in the $I(\mathrm{G}) / I(\mathrm{D})$ ratio, (2) the t-PA peak has transformed from a weak shoulder band at $1130 \mathrm{~cm}^{-1}$ to a well-defined peak at $1180 \mathrm{~cm}^{-1}$ and a large enhancement in overtone feature around $2270 \sim 2600 \mathrm{~cm}^{-1}$, and (3) a stronger Raman intensity in $1100 \sim 1650 \mathrm{~cm}^{-1}$ for $\mathrm{N}$-doped UNCD films is observed at the edge. These observed results strongly suggest that (1) the undoped UNCD films grown at the edge seem to contain a larger percentage of $\mathrm{sp}^{2}$-bonded carbons due to the smaller nanodiamond crystallite clusters (see Figure 4 of AFM images) as compared to that at the center, and (2) the N-doped UNCD films show an increase in clustering and disordering of aromatic $\operatorname{sp}^{2}(\pi)$-bonded carbons in the grain boundaries $[5,6,11]$. However, one should also note that a large increase in Raman intensity of the top two spectra, such as the peak at $1540 \mathrm{~cm}^{-1}$, may in part due to the higher scattering cross-section of $\mathrm{sp}^{2}$ sites for visible Raman transitions.

The nanodiamond crystallinity for both undoped and Ndoped UNCD films has shown to enhance as going from the edge to the center of substrate. This is evidenced by the observation of an increase in Raman intensity ratio $\left(\mathrm{sp}^{3} / \mathrm{sp}^{2}\right)$ of diamond peak (shift from $1350 \mathrm{~cm}^{-1}$ to $1332 \mathrm{~cm}^{-1}$ ) relative to graphite G-peak (shift from $1540 \mathrm{~cm}^{-1}$ to 1560 $1590 \mathrm{~cm}^{-1}$ ). On the other hand, the spectral peaks of t-PA seem to disappear gradually from the edge to the center substrate position, while only a small shoulder around $1130 \mathrm{~cm}^{-1}$ and a broad band around $2600 \sim 2700 \mathrm{~cm}^{-1}$ are observed, suggesting an improvement of $\mathrm{sp}^{2}$-bonded carbons phases in the grain boundaries of UNCD films. Meanwhile, the broad spectral background is also greatly reduced.

The spatial variation in compositional changes of the UNCD films with and without $\mathrm{N}$-doping as shown in Figure 1 is possibly resulted from the varying alpha parameter (appearing of crystal facets) due to the inhomogeneous of plasma density distribution from the perimeter of the plasma ball to the center of substrate that leads to different gas phase composition, varying substrate temperature and surface chemistry for diamond film growth $[15,16]$.

Figure 2 shows the intensity variation of spatially resolved Si Raman peak at $520 \mathrm{~cm}^{-1}$ taken along the radial 


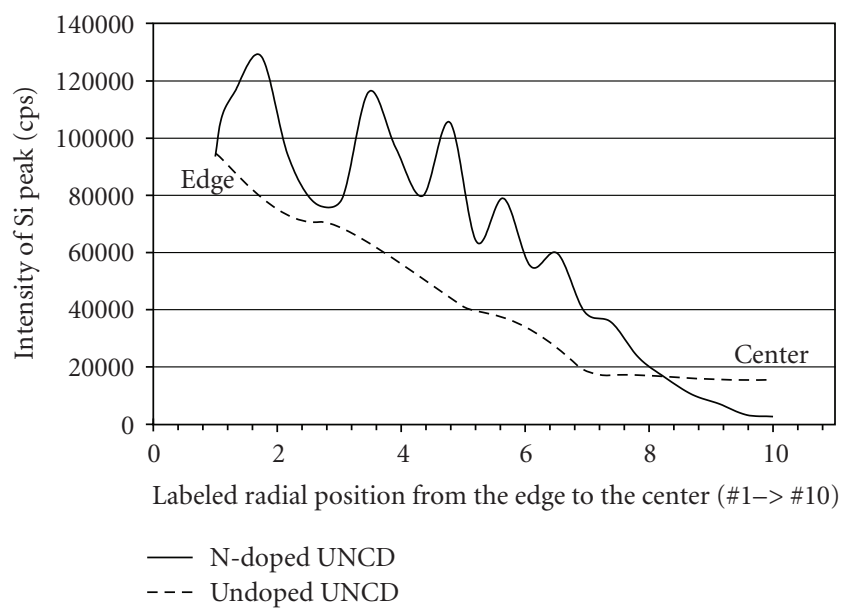

FIGURE 2: Intensity variation of spatially resolved Si Raman peak at $520 \mathrm{~cm}^{-1}$ of UNCD film from the edge to the center position of substrates.

position of substrate from the edge to the center, for both UNCD films with (solid) and without (dash) $\mathrm{N}_{2}$ doping. For comparison, the $\mathrm{Si}$ peak of undoped UNCD film is normalized by referring to that of N-doped UNCD film at the edge position, labeled \#1. The Si peak is observed because the thicknesses of UNCD films are thin enough for being penetrated through by the excitation laser beam. The penetration depth of the visible light at an excitation wavelength of $632.8 \mathrm{~nm}$ is about $1 \sim 2 \mu \mathrm{m}$ [11]. Therefore, the change of Si peak intensity should inversely reflect the thickness uniformity of UNCD films over the Si substrate from the edge to the center. From the results illustrated in Figure 2, the films are thicker and smoother at the center $(\sim 1 \mu \mathrm{m})$ than those at the edge. The oscillation of Si peak intensity for N-doped UNCD film indicates its thickness fluctuation as going from the edge to the center positions, which is much larger than that of undoped UNCD film. The actual thickness profile of the films along the substrate position can be obtained by the calibration of Si Raman peak intensity.

3.2. ATR/FT-IR Spectra of UNCD Films. Figure 3(a) shows ATR/FT-IR absorption spectra of undoped (Blue) and Ndoped (Red) UNCD films at the center position of substrate in the mid-IR range of $675 \sim 4000 \mathrm{~cm}^{-1}$. The absorption of water $\left(3500 \sim 3900 \mathrm{~cm}^{-1}\right.$ and $\left.1400 \sim 1800 \mathrm{~cm}^{-1}\right)$ and carbon dioxide $\left(2350 \mathrm{~cm}^{-1}\right)$ is noticed in the spectra [17]. The C$\mathrm{C}$ and $\mathrm{C}-\mathrm{H}$ rocking characteristics of undoped UNCD films are observed between $800 \sim 1200 \mathrm{~cm}^{-1}$ and $600 \sim 900 \mathrm{~cm}^{-1}$, respectively, in the spectrum (Blue) [13].

For this study, the focus is on the transformation of $\mathrm{sp}^{3}$ to $\mathrm{sp}^{2} \mathrm{CH}$ vibrations upon nitrogen doping that are located in the spectral ranges of $2600 \mathrm{~cm}^{2}$ to $3200 \mathrm{~cm}^{2}$ as shown in Figures 3(b), 3(c). Both N-doped Figure 3(b) and undoped Figure 3(c) spectra are weak, broad, and featureless, so a detailed deconvolution technique of Gaussian fitting and baseline correction of ATR/FT-IR spectra is required to analyze the UNCD films at center of substrate. The tentative

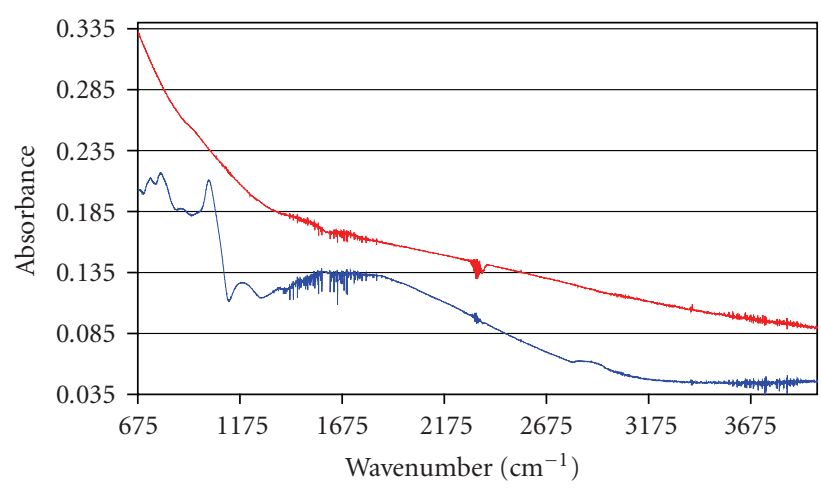

(a)

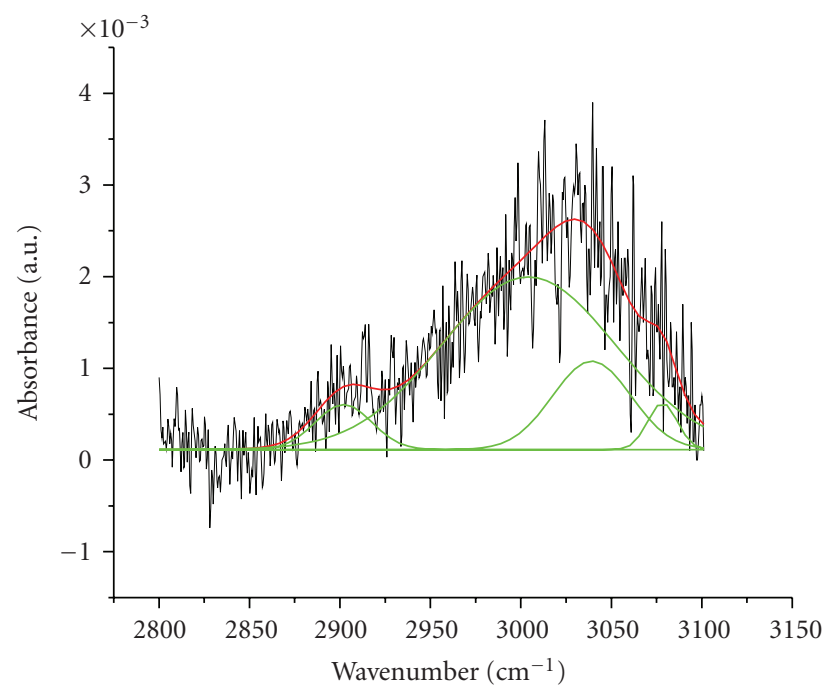

(b)

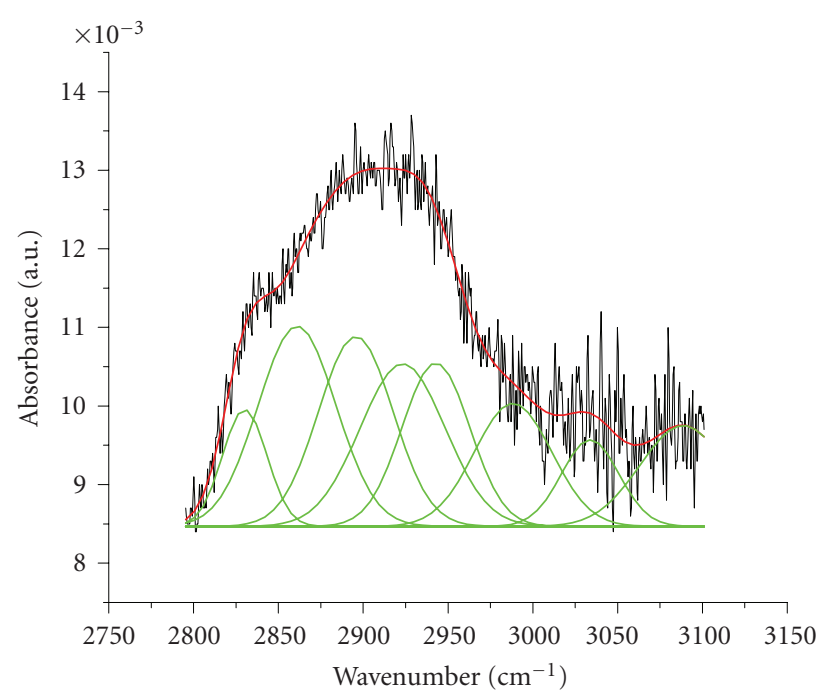

(c)

FIgure 3: (a) ATR/FT-IR spectra of undoped (blue) and N-doped (red) UNCD films by MWCVD at the center position (labeled \#10). (b), (c) Deconvolution (green) of ATR/FTIR spectra of N-doped (top) and undoped (bottom) UNCD films at the center position in $\mathrm{CH}$ vibrational region of $2600 \sim 3200 \mathrm{~cm}^{-1}$ after baseline correction and by Gaussian fitting (red). 
TABLE 1: Spectral band assignment of $\mathrm{CH}_{\mathrm{x}}$ stretching vibration modes of UNCD films at the center position, where minor peaks are shown in parenthesis.

\begin{tabular}{lcc}
\hline $\begin{array}{l}\text { Assignment in [13, } \\
14,16,17] /\left(\mathrm{cm}^{-1}\right)\end{array}$ & $\begin{array}{c}\text { Assignment in experiments } \\
\text { N-doped } \\
\text { UNCD films }\end{array}$ & $\begin{array}{r}\text { Undoped } \\
\text { UNCD films }\end{array}$ \\
\hline $\mathrm{sp}^{2}-\mathrm{CH}_{2}$ (sym.)/2980 & 3004 & $(2988)$ \\
$\mathrm{sp}^{2}-\mathrm{CH}_{2}$ (asym.)/3080 & 3078 & $(3088)$ \\
$\mathrm{sp}^{2}-\mathrm{CH} / 3025$ & 3039 & $(3033)$ \\
$\mathrm{sp}^{3}-\mathrm{CH} / 2900 \sim 2920$ & $(2902)$ & 2895 \\
$\mathrm{sp}^{3}-\mathrm{CH}_{2}$ (sym.)/2850 & - & 2861 \\
$\mathrm{sp}^{3}-\mathrm{CH}_{2}$ (asym.)/2920 & - & 2923 \\
\hline
\end{tabular}

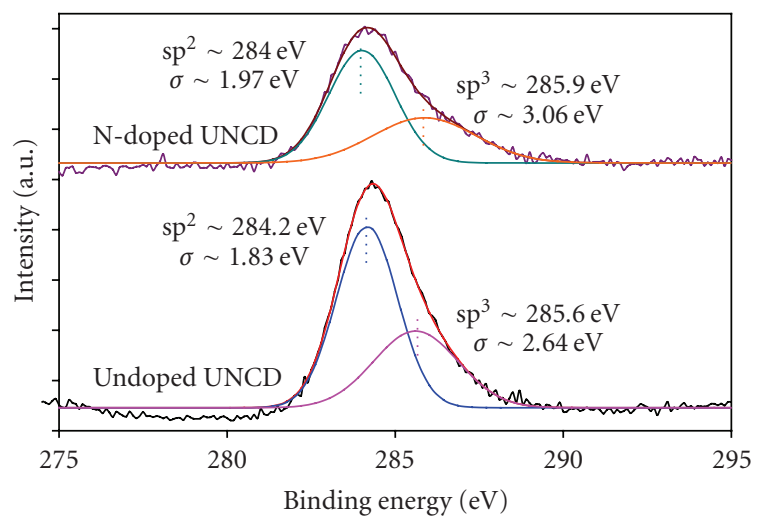

Figure 4: XPS C 1s spectra of undoped (bottom) and N-doped (top) UNCD films with Gauss fits of $\mathrm{sp}^{2} \& \mathrm{sp}^{3}$ peaks.

peak assignments are summarized in Table 1. The distinctive differences between $\mathrm{N}$-doped and undoped UNCD films at center are in the spectral range of $2800 \sim 3100 \mathrm{~cm}^{-1}$. The $\mathrm{sp}^{2}-\mathrm{CH}_{2}$ asymmetric and $\mathrm{sp}^{2}-\mathrm{CH}$ stretching vibrations at $3078 \mathrm{~cm}^{-1}$ and $3039 \mathrm{~cm}^{-1}$ are observed in N-doped UNCD films $[14,18,19]$. The $\mathrm{sp}^{3}-\mathrm{CH}$ and $\mathrm{sp}^{3}-\mathrm{CH}_{2}$ asymmetric stretching vibrations at $2895 \mathrm{~cm}^{-1}$ and $2923 \mathrm{~cm}^{-1}$ are shown for undoped UNCD films $[13,14,18,19]$. We have also analyzed the ATR/FT-IR absorption spectra in the same spectral ranges as in Figure 3(b) for undoped and N-doped UNCD films at the edge position of substrate, and the results are quite similar to those at the center. The only difference is that the $\mathrm{CH}$ vibrational features of UNCD films are stronger and better defined at the center than those at the edge position. These results are in agreement with the Raman spectroscopic observations above that the incorporation of nitrogen into UNCD films would lead to the formation of $\mathrm{sp}^{2}$-bonded carbons.

3.3. X-Ray Photoelectron Spectroscopy. In Figure 4, the XPS C 1s spectra of undoped and N-doped UNCD films at the center position (labeled \#10) of the substrate are deconvoluted by Gaussian multiple peaks fitting into two main components with the binding energies (BEs) of 284.0
$284.2 \mathrm{eV}$ (for carbon $\mathrm{sp}^{2}$ bonding) and 285.6 285.9 eV (for carbon $\mathrm{sp}^{3}$ bonding). In $\mathrm{N}$-doped UNCD films, the following XPS spectral features are observed: (1) $\mathrm{sp}^{2}$ peak shifts to lower BE by $0.2 \mathrm{eV}$, (2) $\mathrm{sp}^{3}$ peak shifts to higher BE by $0.3 \mathrm{eV}$, and (3) both $\mathrm{sp}^{2}$ and $\mathrm{sp}^{3}$ peaks are broadened as compared to the undoped UNCD films. The observed spectral broadening and shifts of $\mathrm{C} 1 \mathrm{~s} \mathrm{BE}$ have been suggested due to the interactions of $\mathrm{C}$ with $\mathrm{N}[20,21]$. The broadened $\mathrm{C} 1 \mathrm{~s}$ peaks in XPS imply the increasing in the formation and disorderness of $\mathrm{sp}^{2}$-bonded carbon phase as resulted from the incorporation of nitrogen into UNCD films that have also been illustrated in Raman and ATR/FT-IR spectra above.

3.4. AFM Morphology of UNCD Films. The AFM surface morphology of UNCD films was monitored for the spatial scanning areas with different film thickness. Figure 5 shows AFM surface morphology of undoped UNCD films (top four) and N-doped UNCD films (bottom four), both scanned at the edge (upper two labeled as (e)) and center (lower two labeled as (c)) positions of substrates.

In Figure 5 (top), the undoped UNCD films were formed by coalescence of coin-like diamond crystal clusters. In a smaller scanning area (or high resolution with a scale bar $100 \mathrm{~nm}$ ) of Figure 5 (top, upper-right), the sizes of diamond crystal clusters at the edge are $433 \mathrm{~nm}$ and $391 \mathrm{~nm}$ as marked by red and blue arrows. In a larger scanning area (or low resolution with a scale bar $1 \mu \mathrm{m}$ ) of Figure 5 (top, lowerleft), the sizes of diamond crystal clusters at the center are $817 \mathrm{~nm}, 701 \mathrm{~nm}$, and $695 \mathrm{~nm}$ as marked by red, green, and blue arrows. In a smaller scanning area (or high resolution with a scale bar $100 \mathrm{~nm}$ ) of Figure 5 (top, lower-right), the radius and central spacing of diamond crystal clusters at the center are about $471 \mathrm{~nm}$ and $527 \mathrm{~nm}$ as marked by green and blue arrows. Therefore, the sizes of diamond crystal clusters increase from $400 \sim 500 \mathrm{~nm}$ (top, upper-left, edge) to $700 \sim$ $800 \mathrm{~nm}$ (top, lower-left, center) along the radial position of substrate.

However in Figure 5 (bottom), the N-doped UNCD films were formed by coalescence of erythrocyte-like. In a larger scanning area (or low resolution with a scale bar $1 \mu \mathrm{m}$ ) of Figure 5 (bottom, left), the sizes of diamond crystal clusters at the edge are $794 \mathrm{~nm}, 915 \mathrm{~nm}$, and $895 \mathrm{~nm}$ as marked by red, green, and blue arrows. The sizes of diamond crystal clusters at the center are $792 \mathrm{~nm}, 724 \mathrm{~nm}$, and $781 \mathrm{~nm}$ as marked by red, green, and blue arrows. In a smaller scanning area (or high resolution with a scale bar $100 \mathrm{~nm}$ ) of Figure 5 (bottom, upper), the widths of the thicker circumferential part of the erythrocyte-like diamond crystal cluster at the edge are about $348 \mathrm{~nm}$ and $189 \mathrm{~nm}$ as marked by green and blue arrows. Therefore, the diamond crystal clusters of $800 \sim 1000 \mathrm{~nm}$ in size over the substrate, both at the edge and center positions, are more uniform than that of undoped UNCD films over the entire substrate areas.

It is worthy of mentioning that the morphology of N-doped UNCD film seems to show a small opening in each diamond crystal cluster at the center as compared to that at the edge as shown in Figure 5 (bottom, lower-left). However, the similar morphology without those openings 


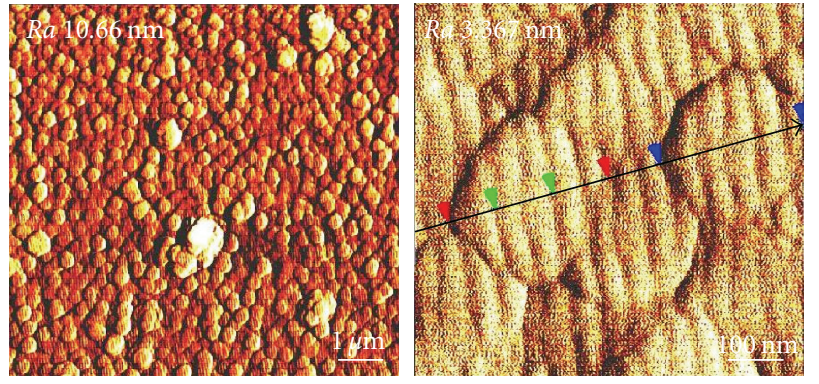

(e)

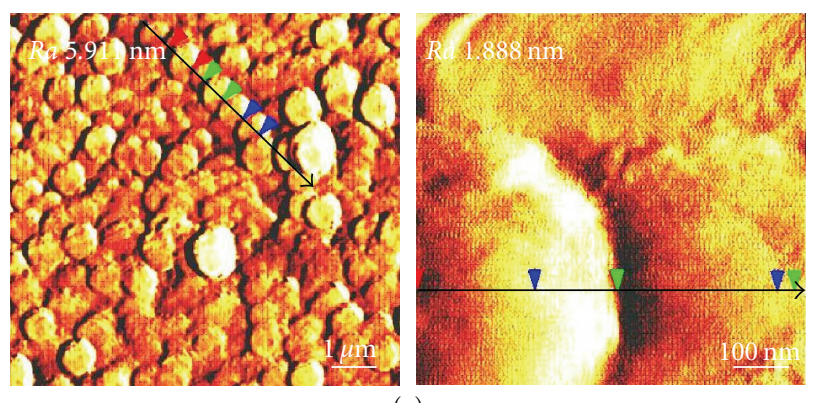

(c)
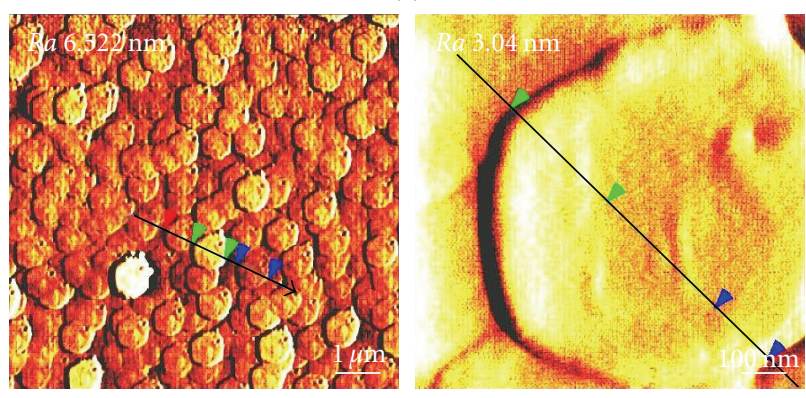

(e)
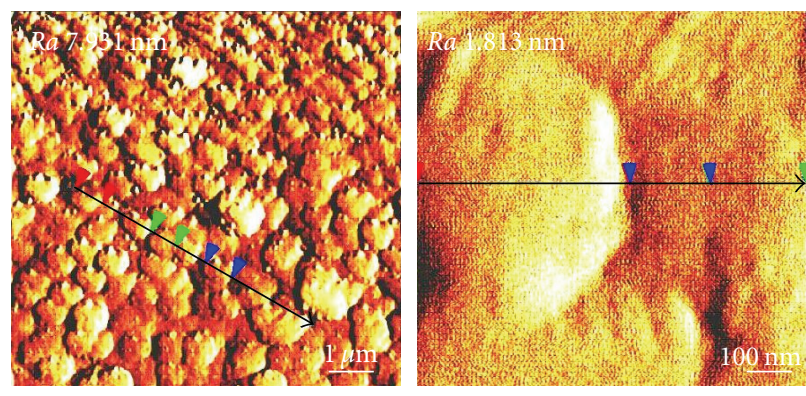

(c)

Figure 5: AFM surface morphology of undoped (top four) and $\mathrm{N}$-doped (bottom four) UNCD films, scanned at edge (upper two labeled as (e)) and center (lower two labeled as (c)) in different areas.

was observed in the AFM image scanned by using a new probe as shown in Figure 6(b), where Figure 6(a) is the same AFM image as Figure 5 (bottom, lower-left). Therefore, those openings are the artifacts coming from the convolution of the tip and sample surface.

In a smaller scanning area (or a higher resolution with a scale bar $100 \mathrm{~nm}$ ) of Figure 5 (top, right two), the surface morphology of each diamond crystal cluster of undoped UNCD films was revoluted from the periodically well-

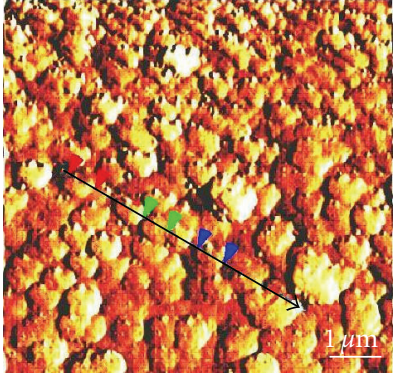

(a)

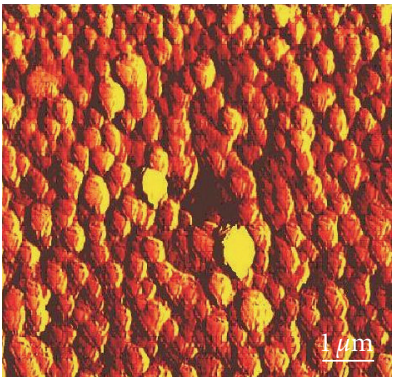

(b)
FIGURE 6: AFM surface morphology of N-doped UNCD films scanned at center by different probes (a) and (b).

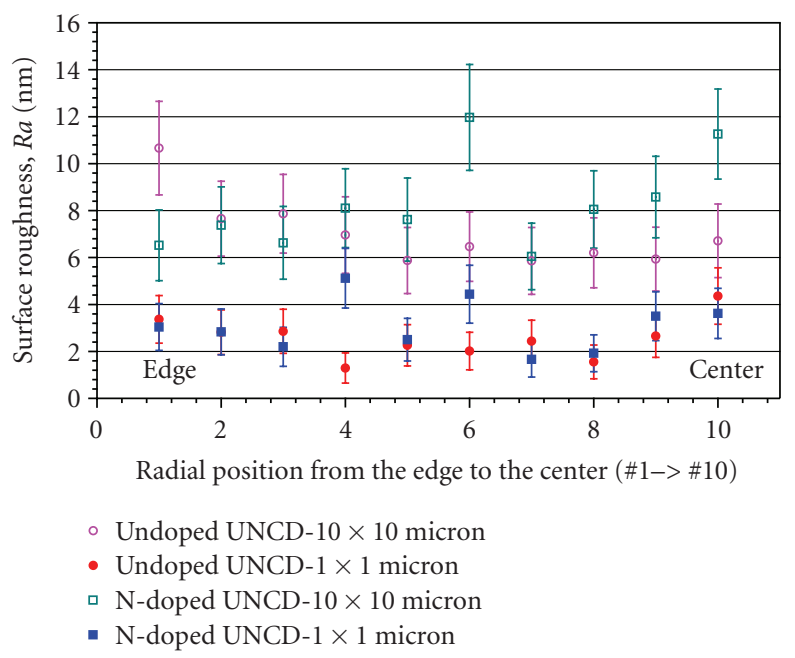

Figure 7: AFM mean surface roughness $(R a)$, with standard deviation error bar, of undoped and N-doped UNCD films scanned at different film areas.

aligned ridges of 70 80 $\mathrm{nm}$ in spacing (two ridges of $155 \mathrm{~nm}$ as indicated by green arrows) at the edge (top, upper-right) into a ballic surface profile at the center (top, lower-right). On the other hand, in Figure 5 (bottom, right two), the surface morphology of each diamond crystal cluster of Ndoped UNCD films was revoluted from a flat surface at the edge (bottom, upper-right) into a bump-like profile at the center (bottom, lower-right).

The observed AFM morphologies may suggest that when undoped UNCD films grow thicker at the center of substrate, the diamond crystal clusters as building blocks have transformed into a 3-dimensional growth, the larger diamond clusters have been grown from a plate-like at the edge into a ball-like at the center of substrate. However, as $\mathrm{N}$-doped UNCD films become thicker, the diamond crystal clusters start with a similar size and then proceed to a preferentially growth in the direction perpendicular to the substrate surface.

Figure 7 shows the mean surface roughness $(R a)$ of the UNCD films for a larger scanning area, $10 \mu \mathrm{m} \times 10 \mu \mathrm{m}$ (pink: undoped UNCD and light green: N-doped UNCD, hollow markers) and a smaller scanning area, $1 \mu \mathrm{m} \times 1 \mu \mathrm{m}$ (red: 
undoped UNCD and blue: N-doped UNCD, solid markers), from the edge (\#1) to the center (\#10) position as labeled in $X$-axis. The standard deviation error bars are indicated together with the mean $R a$ markers.

In pink markers, the $R a$ of the undoped UNCD film gradually decreases from $11 \mathrm{~nm}$ (edge, $\# 1$ ) to around $6 \mathrm{~nm}$ (center, \#10), with the overall mean value of $7 \mathrm{~nm}$ (STED $1.4 \mathrm{~nm}$ ). In red markers, on the other hand, the $R a$ of the undoped UNCD film displays a slight fluctuation with the overall mean $R a 2.5 \mathrm{~nm}$ (STED $0.9 \mathrm{~nm}$ ).

This observation seems to associate with the numbers and size of diamond crystal clusters within the AFM scanning area. For example, in a $(1 \mu \mathrm{m} \times 1 \mu \mathrm{m})$ viewing area, the $R a(2.5 \mathrm{~nm})$ is quite uniform within the area down to $1 \sim 4$ diamond crystal clusters. For a larger viewing of undoped UNCD film, for example, $10 \mu \mathrm{m} \times 10 \mu \mathrm{m}$ area covering about $100 \sim 400$ diamond crystal clusters, the $R a$ increases up to $7 \mathrm{~nm}$. The corresponding standard deviation of the mean $\mathrm{Ra}$ also increases in $10 \mu \mathrm{m} \times 10 \mu \mathrm{m}$ area as compared to $1 \mu \mathrm{m} \times$ $1 \mu \mathrm{m}$ area. The $R a$ is $6 \mathrm{~nm}$ at the center (\#10) with a cluster size of $800 \mathrm{~nm}$ and the $R a=11 \mathrm{~nm}$ in the edge (\#1), while the cluster size is $500 \mathrm{~nm}$, as shown in Figure 5 (top).

Similarly, in light green and blue markers, the overall mean $R a$ of the N-doped UNCD films, both in $10 \mu \mathrm{m} \times$ $10 \mu \mathrm{m}$ and $1 \mu \mathrm{m} \times 1 \mu \mathrm{m}$ scanning areas, is $8 \mathrm{~nm}$ (STED $1.8 \mathrm{~nm}$ ) and $3 \mathrm{~nm}$ (STED $1.1 \mathrm{~nm}$ ), respectively. But the $R a$ of $\mathrm{N}$-doped UNCD films seems to fluctuate larger than that of undoped UNCD films, suggesting the formation of a larger diamond crystal cluster size for the N-doped UNCD films.

3.5. Effect of N-Doping on UNCD Films. Normally, UNCD films grown by MPCVD using $\mathrm{CH}_{4}$ and $\mathrm{Ar}$ gas mixtures without the addition of $\mathrm{N}_{2}$ gas should follow the $\mathrm{C}_{2}$ based growth mechanism [7], where the grown UNCD films should compose of nanocrystalline diamond grains and a structurally disordered mixture of $\mathrm{sp}^{3} / \mathrm{sp}^{2}$-bonded carbons in the grain boundaries. Upon introducing nitrogen into UNCD films, the most energetically favorable sites should be at the grain boundaries, where the lone pair electrons in nitrogen can facilitate the transformation of tetrahedral coordinated sites of $\mathrm{sp}^{3}$-like carbon configuration into a three-fold-coordinated site of $\mathrm{sp}^{2}$-like carbon arrangement with a perturbed $\mathrm{N}$-doped characteristic at the grain boundaries [6]. Thus, the incorporation of nitrogen into UNCD films could lead to an increase in amorphous $\mathrm{sp}^{2}$ bonded $(\pi-)$ carbon phase by the formation of conjugated networking of the chain-like $\mathrm{sp}^{2}$ and $\mathrm{sp}^{1}$ hybridized with $\mathrm{N}$ doped structures together with aromatic carbon clusters in the grain boundaries as evidenced by the results of Raman, ATR/FT-IR, and XPS spectral investigations.

The $\mathrm{sp}^{3}$ nanodiamond grains of undoped UNCD films are electrically inactive based on the theoretical tightbonding calculations [8]. For N-doped UNCD film, the structural transformation has shown to lead to an increase of $\mathrm{sp}^{2}$-carbon phase at the grain boundaries as illustrated in the spectroscopic investigation above that can give the n-type electrical conductivity as resulted from the increase in the electron delocalization of $\pi-\pi^{*}$ transition states. This structural assessment is in agreement with the observed high conductivity (up to $150 \Omega^{-1} \mathrm{~cm}^{-1}$ ) for the nitrogen containing UNCD films, via the enhanced $\mathrm{sp}^{2}$-carbon phase about $\sim 10 \%$ at the dense and connected grain boundaries $[1,21]$.

AFM surface morphology pictures of N-doped UNCD films in Figure 5 show larger nanocrystalline diamond clusters and more wrinkled surface than those of undoped UNCD films. The increase of the overall grain boundary volume and grain size in N-doped UNCD films measured at the center, and the edge positions of substrate could offer a possible film growth mechanism that the $\mathrm{N}$-doped molecular species may reduce secondary nucleation rate by blocking potential sites for $\mathrm{C}_{2}$ addition through the absorption of these $\mathrm{N}$-doped molecules and thus enhance the growth of diamond crystals $[4,6]$.

\section{Conclusions}

The incorporation of nitrogen into UNCD films prepared by MPCVD using $\mathrm{CH}_{4} / \mathrm{N}_{2} / \mathrm{Ar}$ gas mixtures has shown to lead to lower $\mathrm{sp}^{3} / \mathrm{sp}^{2}$-bonded carbon characteristic as evidenced in the spectral features of $\mathrm{sp}^{2}$-bonded carbon phases in the grain boundaries of N-doped UNCD films. Higher amorphous and $\mathrm{sp}^{2}$ carbon feature in thin UNCD film deposited at the edge position of substrate was observed. The increase in size of nanocrystalline diamond clusters leading to a larger fluctuation of surface roughness was also revealed by AFM surface morphology of N-doped UNCD films. The assessment of structural transformation of UNCD films upon nitrogen doping is in agreement with the previously proposed high electron transport via grain boundaries for $\mathrm{N}$ doped UNCD films.

\section{Acknowledgments}

This work was supported by Kinik Company in Taiwan and Institute for Nano Science, Engineering and Technology of Northern Illinois University, USA. The authors would like to thank Dr. Dieter M. Gruen and Dr. Paola Bruno of Argonne National Lab for providing UNCD film samples and valuable discussions, and Dr. Haji-Shiekh for technical assistance on AFM measurement.

\section{References}

[1] O. A. Williams, T. Zimmermann, M. Kubovic, et al., "Electric properties and applications of ultrananocrystalline diamond," in Synthesis, Properties and Applications of Ultrananocrystalline Diamond, D. M. Gruen, O. A. Shenderova, and A. Ya. Vul', Eds., vol. 192 of NATO Science Series II: Mathematics, Physics and Chemistry, pp. 373-382, Springer, Berlin, Germany, 2005.

[2] D. M. Gruen, P. C. Redfern, D. A. Horner, P. Zapol, and L. A. Curtiss, "Theoretical studies on nanocrystalline diamond: nucleation by dicarbon and electronic structure of planar defects," The Journal of Physical Chemistry B, vol. 103, no. 26, pp. 5459-5467, 1999.

[3] X. Xiao, J. Birrell, J. E. Gerbi, O. Auciello, and J. A. Carlisle, "Low temperature growth of ultrananocrystalline diamond," Journal of Applied Physics, vol. 96, no. 4, pp. 2232-2239, 2004. 
[4] Q. Chen, G. M. Swain, and D. M. Gruen, "Effects on $\mathrm{N}_{2}$ in $\mathrm{CH}_{4} / \mathrm{Ar}$ plasma on the microstructure and electrochemical properties of ultrananocrystalline diamond films," in Proceedings of the 198th Electrochemistry Society Symposium on Electrochemistry of Carbon Materials, vol. 34, pp. 73-82, Phoenix, Ariz, USA, October 2000.

[5] P. Zapol, M. Sternberg, L. A. Curtis, T. Frauenheim, and D. M. Gruen, "Tight-binding molecular-dynamics simulation of impurities in ultrananocrystalline diamond grain boundaries," Physical Review B, vol. 65, no. 4, Article ID 045403, 11 pages, 2002.

[6] J. Birrell, J. E. Gerbi, O. Auciello, J. M. Gibson, D. M. Gruen, and J. A. Carlisle, "Bonding structure in nitrogen doped ultrananocrystalline diamond," Journal of Applied Physics, vol. 93, no. 9, pp. 5606-5612, 2003.

[7] V. I. Polyakov, A. I. Rukovishnikov, N. M. Rossukanyi, et al., "Charge-based deep level transient spectroscopy of undoped and nitrogen-doped ultrananocrystalline diamond films," Diamond and Related Materials, vol. 12, no. 10-11, pp. 1776-1782, 2003

[8] I. S. Beloborodov, P. Zapol, D. M. Gruen, and L. A. Curtiss, "Transport properties of $n$-type ultrananocrystalline diamond films," Physical Review B, vol. 74, no. 23, Article ID 235434, 5 pages, 2006.

[9] J. Birrell, J. E. Gerbi, O. Auciello, J. M. Gibson, J. Johnson, and J. A. Carlisle, "Interpretation of the Raman spectra of ultrananocrystalline diamond," Diamond and Related Materials, vol. 14, no. 1, pp. 86-92, 2005.

[10] D. M. Gruen, "Nanocrystalline diamond films," Annual Review of Materials Science, vol. 29, pp. 211-259, 1999.

[11] R. Arenal, G. Montagnac, P. Bruno, and D. M. Gruen, "Multiwavelength Raman spectroscopy of diamond nanowires present in $n$-type ultrananocrystalline films," Physical Review $B$, vol. 76, no. 24, Article ID 245316, 6 pages, 2007.

[12] R. Arenal, P. Bruno, D. J. Miller, M. Bleuel, J. Lal, and D. M. Gruen, "Diamond nanowires and the insulator-metal transition in ultrananocrystalline diamond films," Physical Review B, vol. 75, no. 19, Article ID 195431, 11 pages, 2007.

[13] N. Aggadi, C. Arnas, F. Benedic, et al., "Structural and chemical characterization of soot particles formed in $\mathrm{Ar} / \mathrm{H}_{2} / \mathrm{CH}_{4}$ microwave discharges during nanocrystalline diamond film synthesis," Diamond and Related Materials, vol. 15, no. 4-8, pp. 908-912, 2006.

[14] P. John, D. K. Milne, I. C. Drummond, J. I. Wilson, M. G. Jubber, and J. A. Savage, "Attenuated total reflection infrared absorption in CVD diamond films," in Diamond Optics V, vol. 1759 of Proceedings of SPIE, pp. 209-217, San Diego, Calif, USA, July 1992.

[15] Private discussion with Dr. D. M. Gruen, Argonne National Lab, Argonne, Ill, USA.

[16] Y. Yokota, Y. Ando, K. Kobashi, T. Hirao, and K. Oura, "Morphology control of diamond films in the region of $\alpha=$ 1 - 1.5 using a $60-\mathrm{kW}$ microwave plasma CVD reactor," Diamond and Related Materials, vol. 12, no. 3-7, pp. 295-297, 2003.

[17] P. Thongnopkun and S. Ekgasit, "Attenuated total reflection Fourier transform infrared spectra of faceted diamonds," Analytica Chimica Acta, vol. 576, no. 1, pp. 130-135, 2006.

[18] K. M. McNamara, B. E. Williams, K. K. Gleason, and B. E. Scruggs, "Identification of defects and impurities in chemicalvapor-deposited diamond through infrared spectroscopy," Journal of Applied Physics, vol. 76, no. 4, pp. 2466-2472, 1994.
[19] E. J. Correa, Y. Wu, J.-G. Wen, R. Chandrasekharan, and M. A. Shannon, "Electrical conduction in undoped ultrananocrystalline diamond thin films and its dependence on chemical composition and crystalline structure," Journal of Applied Physics, vol. 102, no. 11, Article ID 113706, 10 pages, 2007.

[20] C. Ronning, H. Feldermann, R. Merk, H. Hofsäss, P. Reinke, and J.-U. Thiele, "Carbon nitride deposited using energetic species: a review on XPS studies," Physical Review B, vol. 58, no. 4, pp. 2207-2215, 1998.

[21] P. Achatz, O. A. Williams, P. Bruno, D. M. Gruen, J. A. Garrido, and M. Stutzmann, "Effect of nitrogen on the electronic properties of ultrananocrystalline diamond thin films grown on quartz and diamond substrates," Physical Review B, vol. 74, no. 15, Article ID 155429, 7 pages, 2006. 

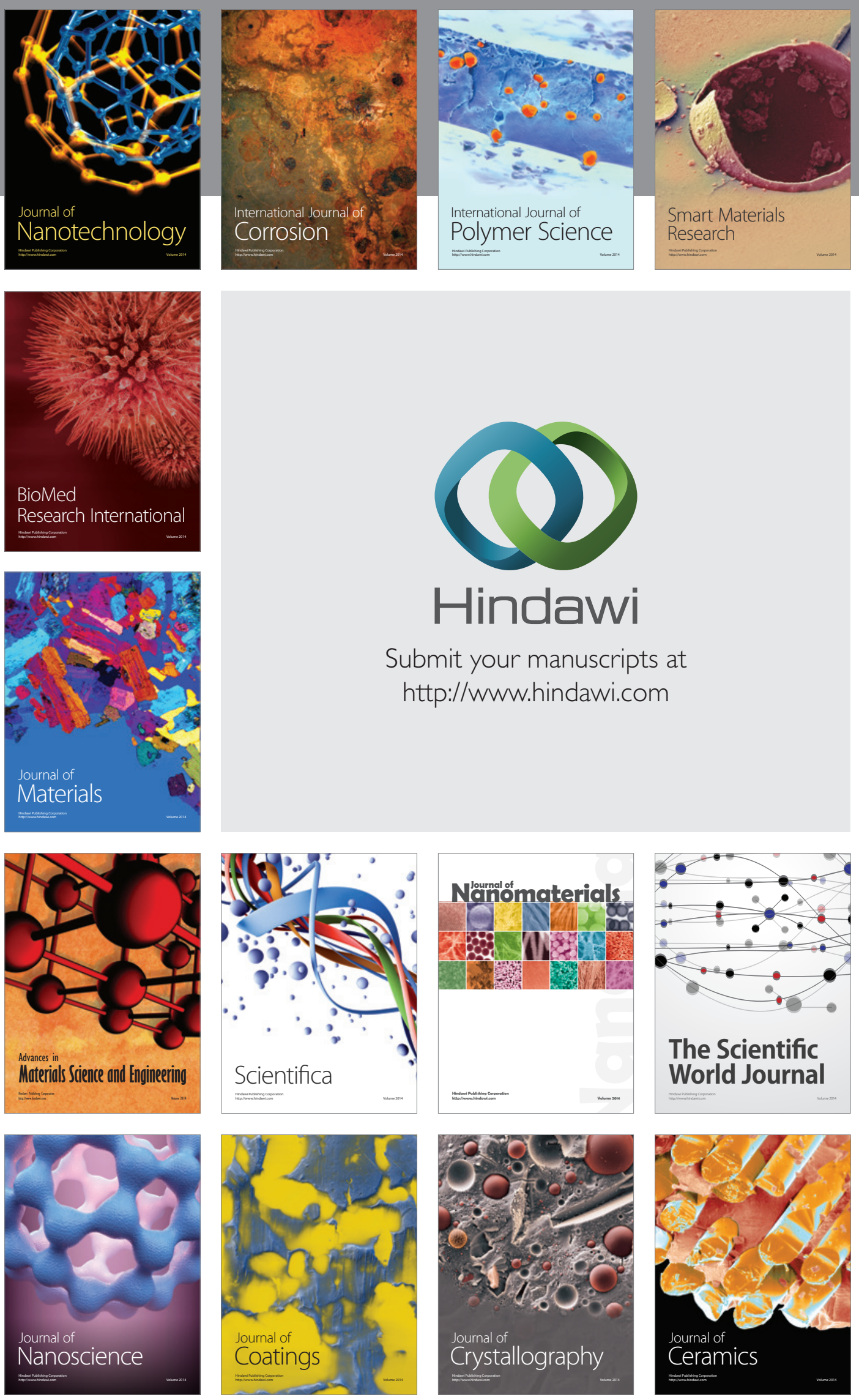

The Scientific World Journal

Submit your manuscripts at

http://www.hindawi.com

\section{World Journal}

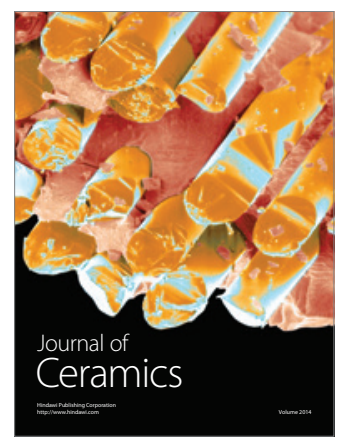

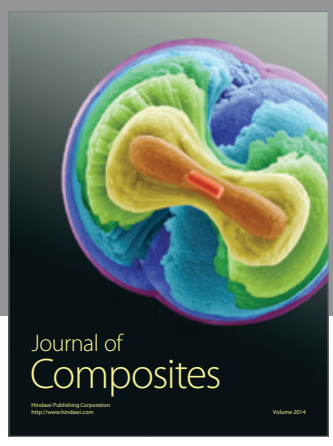
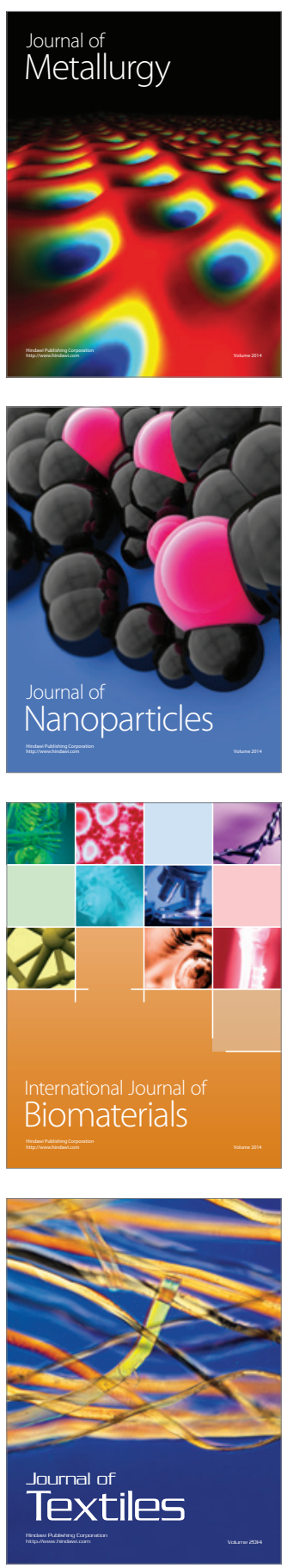